IJMS 2016 vol. 4 (1): 40-47

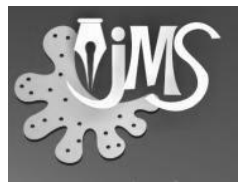

International Journal of Multidisciplinary

Studies (IJMS)

Volume 4, Issue 1, 2017

DOI: http://doi.org/10.4038/10.4038/ijms.v4i1.35

\title{
Legal and Engineering Aspects of Deployable Black Boxes with Video Recording Capability
}

\author{
Abeysekara Thusitha $\mathrm{B}^{1}$ and Abimani Menuwansala Peiris $\mathrm{H}^{2}$ \\ ${ }^{1}$ Department of Civil Law, Faculty of Law, General Sir John Kotelawala Defence University, Sri \\ Lanka \\ ${ }^{2}$ Department of Aeronautical Engineering, Faculty of Engineering, General Sir John Kotelawala \\ Defence University, Sri Lanka
}

\begin{abstract}
Black box is a compilation of data produced by on-board and ground communications, specifically and inherently in digital nature; therefore it is a digital database and part of a discussion on protection of digital databases. The Existing Black box technology used in Commercial Aircraft is accommodated with Affixed type black boxes. However, if the Deployable black boxes are implemented with fixed black boxes as a dual combined recorder system, the discovery of crash location can be determined with more effectually than the present technology. The lives of the survivors, initiation of aircraft accident and investigation progression obtain safety recommendations to avoid similar happenstances will depend upon the accessibility to the crash location immediately by search and rescue organizations with the reliable information they achieved through satellite communications. Deployable Recorders will provide a backup source of flight data other than the fixed black box data; assist the investigators to carry out their investigations with more trustworthy information while implementing the video recording capability. A new idea has been introduced in this research to implement a mechanism to deploy a parachute with the ejection of the deployable recorder from the aircraft to minimize the impact during its dwindling to the ground. Not only in engineering but also the legal aspects of Deployable black boxes work along with the rights of the human beings. The legal aspect of the Deployable black boxes connect with aircraft accident and an incident investigation process can be discussed in the preview of domestic and international law; for example under the Convention on International Civil Aviation. Annex 13 of the Chicago Convention is endowed with the international requirements for the investigation of aircraft accidents and incidents. The protection over digital databases can be achieved through legal and technological means (Technological Protection Mechanisms-TPMs). The paper investigates the legal and engineering aspects of Black box technology used in Commercial Aircraft.
\end{abstract}

KEYWORDS: Black Box, Deploy, Chicago Convention, Databases, International Air Law

Corresponding author: Thusitha B. Abeysekara, email: thusitha313@yahoo.com 


\section{INTRODUCTION}

\section{Dialectical campaigning on engineering and law}

The law governs every aspects of human life. The law touches the human pulse and teeth of gear wheels. Therefore, it is not an accident to discuss the legal aspect of engineering or vice versa. Engineering ensures the smooth running of human life, while law keeps it on the right track. Aeronautical engineering is not only the subject which discusses the way of materials bring to the air, but also deals with responsibilities of transport passengers and goods, as well as equipment. International Air Law works with many aspects such as safety of passengers, responsibilities of transportation, airport regulations, air pollution, hijacking and areal warfare (Abeyratne, 2016). Therefore, the two subjects, law and engineering, work for the betterment of human being at large.

\section{Deployable Black boxes}

Black box is contructed with bright orange or bright yellow which is capable to withstand fire, loads and impacts during a crash. Presently, a fixed type of black boxes are used in commercial airliners, though it is recommended to implement a combined system of deployable black box and fixed type black box together, after aircraft accidents such as Malaysian Airlines MH 370, Air France 447, Adam Air KI 574. This reliable fixed type black box device is contains a FDR (Flight Data Recorder), CVR (Cockpit voice Recorder) and ULB (Under water Location Beacon).

In this design, the location beacons are directing the notification with a nominal operating frequency of $37.5 \mathrm{kHz}$ throughout a particular duration. If the battery of the ULB is dead, it will not receive any signals. Therefore, the SAR (Search and Rescue) process should be accomplished with the co-relation of the battery duration. Deployable Recorders which is a combination of FDR, CVR and ELT
(Emergency Location Transmitter) were recommended for commercial aircrafts by Flight Safety Foundation, which is a technology used by some Military aircrafts. The ELT device, which is comprised in deployable black boxes can immediately send precise, relevant information through satellite communications to SAR organizations, to activate the search and rescue processes immediately to rescue the survivors at the crash site. Then the immediate aircraft accident and incident investigation process can commence.

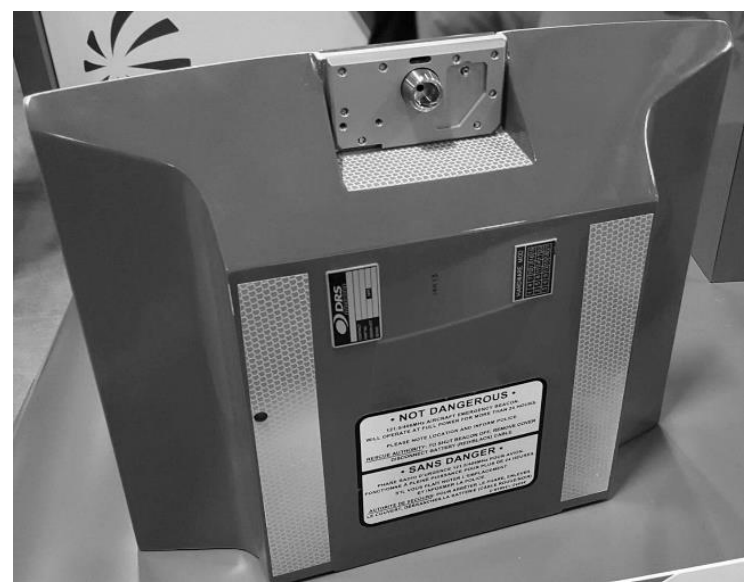

Figure 1. Automatic Deployable Recorders, Photo courtesy goes to DRS Technologies, available at http://prov.in.ua /images/ users/ Image/ADFR.jpg igure Automatic Deployable Recorders, Figure courtesy goes to DRS Technologies, available at http://prov.in.ua/images/users/Image/ADFR.jpg

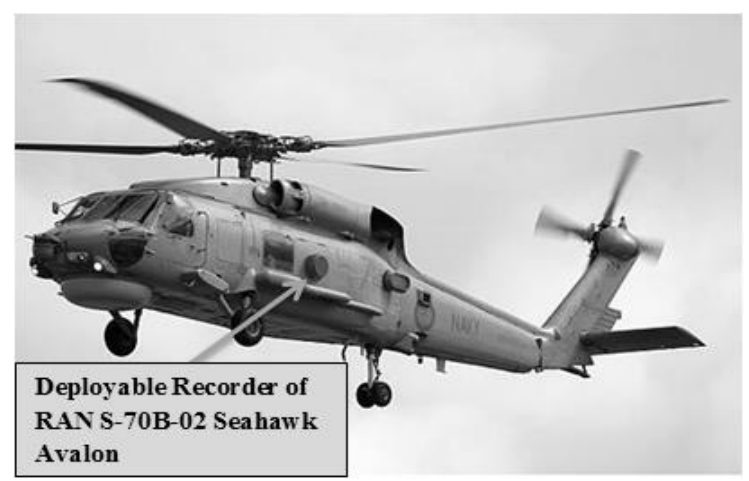

Figure 2. Automatic Deployable Recorders used by RAN S-70B-2 Seahawk Avalon, Photo courtesy goes to wikimedia, available at 
https://upload.wikimedia.org/wikipedia/commo ns/thumb/1/1c/RAN_S-70B

2_Seahawk_Avalon_2011.jpg/440px-RAN_S70B-2_Seahawk_Avalon_2011.jpg

The legal aspect of the above, especially aircraft accident and incident investigation process, can be discussed in the preview of the Convention on International Civil Aviation, signed in Chicago, on 7 December 1944 (Chicago Convention) and its Annex 13- Aircraft Accident and Incident Investigation. Annex 13 states that the objective of the investigation of an accident or incident is prevention. To work out this, the root causes of an aircraft accident or serious incident must be identified in order to prevent repeated occurrences. The identification of causal factors is best accomplished through a properly conducted investigation. Annex 13 of the Chicago Convention provides the international requirements for the investigation of aircraft accidents and incidents.

In turn, in engineering, the Deployable recorder is designed to release in advance the crash which is predetermined by several sensors. A predictable design for deployable recorders is associated with an airfoil profile which will provide a lifting force, as well as an aid for floating if it falls to some aquatic area. Unlike the the existing black box, if the deployable recorder system will originate into use as a combined system with fixed black box and deployable black box, the search and rescue process will be more convenient, time saving, cost saving, effective and efficient. In addition, relevant information will be transmitted to SAR authorities immediately when the deployable recorder is being ejected by satellite communication.

\section{a. CVR}

The CVR records voice communications transmitted from or received in the airplane by radio, voice communications of flight crew members on the flight deck, voice communications of flight crew members on flight deck using the aircraft's interphone system, navigational aids or approach aids towards headset or speaker, voice communications of flight crew members using the passenger loudspeaker system, audio alerts and other ambient sounds in the cockpit through microphones inside the cockpit. An associated control unit is used for the process of preamplification before the audio signals transferred to the CVR. Approximately, the CVR records last 2 hours of audio data. Magnetic tape was the medium of recording in previously analogue recorders. Currently digitalized voice data are being recorded in memory chips in modern solid- state CVRs.

At this juncture, it is worth to keep in mind that the above points are highly sensitive topics in legal aspects of information. Widely this argument can be placed in Information Law which deals with the balancing process of public information and private information. If there is no public interest with the information then there is privacy. Recorded voice communications transmitted from or received in the airplane by radio, recorded voice communications of flight crew members on the flight deck, voice communications of flight crew members on flight deck using other means, voice communications of flight crew members using the passenger loudspeaker system or audio alerts and other ambient sounds in the cockpit through microphones inside the cockpit are part of information which can be considered as privacy issue relating to the flight crew member. Any member can claim his/her privacy right over the CVR information as it emits privacy concerns.

The right to privacy was coined in legal literature by Samuel Warren and Louis Brandeis in their essay published in the Harvard Law Review in 1890 (Millard, 2005), before being invented of powered aircraft by Wright Brothers. The privacy is a right which flies beyond the jurisdictions like aircraft. While the vast majority of countries in the world do not yet have data protection laws, a number of jurisdictions either have general privacy rights, 
sometime entrenched in a constitution, or have sector-specified privacy rules that have an impact on communications (Millard, 2005 p381).

\section{b. FDR}

FDR is a design to record all readings of airspeed, altitude, and heading and the corresponding readings of the first pilot's instruments. Federal Aviation Administration (FAA) has minimum requirement to record 11 to 29 parameters in FDR in the commercial airlines depending on the size of the aircraft. 25 hours of data can be interpreted from a FDR of Deployable Black box. Formerly a magnetic tape was the medium that was used in FDRs as same as in the early CVRs. The technology is now being developed to adopt with memory chips in modern solid-state FDRs, in order to store digitalized information with less space and with a maximum storage.

\section{c. ELT}

The Deployable Black Boxes includes with ELT device which provides the most important information to the SAR organizations to reach the crash location with no adjournment. ELT sends immediate information about tail number of the aircraft, country of origin, the separation location of the deployable recorder with the aircraft, and location of the deployable black box whether it is on water or land, excluding the location of ETOPS (Extended Range Operation with Two-Engine Airplanes) and polar routes to SAR organizations through COSPAS-SARSAT.

\section{Video Recording in Black box}

If the system is enhanced to record the videos on cockpit area, an addition to the recording voice and flight data in the respective recorders, the investigations will be more convenient and less time consuming when investigating the cause of action. Image recorders record images of all flight crew work areas, including instruments and controls. If the cameras were installed inside the cockpit, loss of controls of instruments and display units, hijacking instances, pilot's stress and his decisions which are taken at an emergency can be recorded on the FDR which will lead to an immediate and consistent accident investigation. Video recorders are not installed in commercial airliners due to the pilots' privacy issues.

\section{Fighter Seat Chute deployment Mechanism}

In Fighter aircraft when the pilot seat is ejected, due to the parachute deploys and parachute descent, the pilot may have injuries such as cervical fracture or strain, muscle sprains, cervical vertebrae dislocation, facial fractures, contusions, lacerations severe pain and hemorrhages. In the technology of the ACES II (The Advanced Concept Ejection Seat) which is used in fighter aircraft such as A-10, F-15 and F16 will deploy the chutes according to the mode activation. This activation is independent from aircraft system and performed by environmental sensors which determine the airspeed and altitude conditions. Depending upon the ejection, recovery parachute or drogue chute is deployed according to the mode.

\section{LITERATURE SURVEY}

\section{Implementation of deployable recorders in commercial transport}

If the Deployable Recorders are accommodated with the affixed type of black boxes, this dual combined recorder system will enhance the efficiency of discovering the crash location immediately after an aircraft accident. P. Robert Austin (1999) conducted a study and revealed that, adopting the combined technology of Deployable recorders and fixed recorders to the aircraft structure could provide unrivaled survival information to the investigators.

Linda Werfelman (2009) also noted that implementation of deployable recorders of the currently existing data recording system of 
aircraft and transmitting flight data to the ground stations would be the two alternatives that can be initiated in commercial aircraft to acquire the black box data for the accident investigations.

Designs of different types of recorders can be recognized as deployable recorders, image recorders and combined recorders, according to the fact sheet of the Australian Transport Safety Bureau (2014). This fact sheet suggests the significance of implementing automatic deployable recorder technology, which is already adopted in some military aircraft and image recorder capability to the commercial passenger aircraft during the occurrence of an aircraft accident.

LBlake van den Heuvel - DRS Technologies Canada Ltd. (2014) have presented from his study that automatic deployable flight recorders corresponding to fixed recorder in a DualCombi installation are intended to overcome challenges of remote location crashes which will contribute to recovery of full flight information. Also, he has suggested that automatic deployable recorders are designed to survive a crash in a contrast manner than affixed black box systems. A key way to prevent aircraft accidents can be achieved through deployable flight recorder systems. When the flight Information and voice data can be investigated immediately after a crash, the safety recommendations can be announced to preclude similar accidents and incidents. These stimulating ideas are strappingly strengthened by the Flight Safety Foundation (2014).

Bonsor, K. and Chandler, N. (2001) have mentioned in their study that NTSB is attempting to initiate the capability of video recording of cockpit inside the black box for years, as an improvement in the black box technology to investigate the crash scenario with more facts. Moreover, this study confirmed that, pilots refuse this decision, as it will violate their privacy and further state that available flight data is ample to carry out investigations.

\section{RSEARCH METHODOLOGY}

The study is based on secondary data. The secondary data has been collected from various research articles on engineering and law and published PhD Thesis. Primary data has been collected from International Air Law Conventions such as Convention on International Civil Aviation, signed at Chicago (Chicago Convention) and The Geneva Conventions of 1949 and their Additional Protocols , its Annex 13- Aircraft Accident and Incident Investigation and Convention for The Suppression of Unlawful Acts Against the Safety of Civil Aviation, Signed at Montreal, on 23 September 1971(Montreal Convention 1971), European Union Directives such as Council Directive/Database 96/9 EC (OJ L 077/20), Legal Statutory instruments. As this is a research on legal aspect of an engineering component, a qualitative research approach is employed to lay down the arguments of the study. Therefore, this research was completed using the critical analysis method and qualitative method, both are widely used in legal research. Hence, Scientific method will be the theme line of the work. This refers to a standardized set of techniques for building scientific knowledge, which explain the building of valid observations, interpretation of results, and finally generalization of those results. The scientific method allows researchers to independently and impartially test pre-existing theories and prior findings, and subject them to open debate, modifications, or enhancements (Bhattacherjee, 2012).

\section{RESULTS AND DISCUSSION}

\section{If the deplorable black boxes deployed by manually by the pilot's decision}

The Deployable black boxes automatically eject with the activation of crash sensors with the suspicion occurrence of an accident. After releasing the black box, it will adopt a free flight and falls to the land or sea area. If the pilot is 
permissible to take the decision of "when this ejection of the deplorable black box should be", it will be more precise and realistic, as the human brain is the most advanced and reliable device than the other machineries. If the pilot is unable to call to mind to take this decision with the anxious condition he is facing, then the deployable black box should be ejected automatically with the activation of the crash sensors.

\section{If the deployable black boxes deployed and the harm can cause to human being, any animal or for a property}

If the chute deployment mechanism is introduced to deployable black box technology which is currently using in fighter seat ejection mechanism in fighter jets, there may be a less injury or damage to human beings, animals, or property during an ejection. If there is a system to sense the environmental conditions such as altitude and airspeed, which is related to the deployable black box, the chute can be deployed immediately with the ejection of black box. This deployment mechanism will lead to cause a less destruction due to the colliding impact which the deployable black box can create during a dwindling.

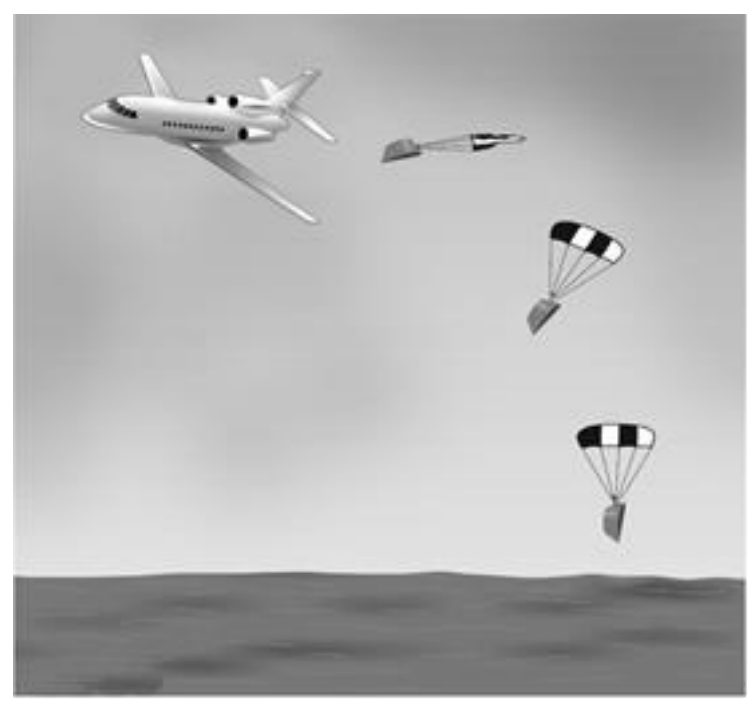

Figure 3. Proposed Chute mechanism ought to implement in Deployable Recorders
Law of war provides the base for the legality of this point. International Humanitarian Law is "a set of rules which seek, for humanitarian reasons, to limit the effects of armed conflict. It protects persons who are not or are no longer participating in the hostilities and restricts the means and methods of warfare" (ICRC, 2004). Article 46 of the Hague Convention on Land Warfare of 18th October 1907 stipulates that private property must be respected and cannot be confiscated by a foreign power (Biehler, 2007).

Further, Rule 9 of the customary International Humanitarian Law study recalls that civilian objects are "all objects that are not military objectives," while Rule 10 recalls that civilian objects are protected against attack, "unless and for such time as they are military objectives." Those rules are applicable in international and non-international armed conflicts (Médecins Sans Frontières, 2016). Articles 12, 21 of Additional Protocol I of Geneva Conventions state that vehicle and other transport are protection concerns objects under International Humanitarian Law.

\section{Black box as a database}

Black box is a compilation of data specifically and inherently in digital nature; therefore it is a digital database. Consequently the database protection is a main legal concern relating to the legal nature of information in the black box. Protection of digital databases can be divided into two separate parts; namely, legal protections and technological protection measures (TPM) (Abeysekara, 2013). 'Database' is a term with no precise definition, and this is certainly the case in the digital context where the black box correctly fixes. As D Lanzotti and D Ferguson state: "a database may be described as an organized collection of data, which is probably, but not necessarily, electronic in nature" (2006). Article 1(2) of Council Directive 96/9 EC (OJ L 077/20) defines that a database is also a "collection of independent works, data or other materials that are arranged in a systematic or methodical way 
and are individually accessible by electronic or other means". In terms of legal protection it is worth to identify the nature of investment in digital databases and to provide protection for the same. The investment in digital databases can be financial or non-financial or both (Abeysekara, 2013). Investment in databases is a reference to the ways in which data is collected, verified, classified and presented in databases. The current legal framework for digital databases are made up of copyright law (for example Copyright, Patents and Designs Act 1988 sections 3(1)(d), 3A), contract law (for example Council Directive 96/9 EC (OJ L 077/20) section 3), sui generis right protection (for example Council Directive 96/9 EC (OJ L 077/20) chapter II) and unfair competition law or misappropriation (for example Council Directive 96/9 EC (OJ L 077/20) section 13). Arguably, these legal regimes provide protection for investment in database creation.

\section{CONCLUSION/ FUTURE WORK}

SAR organizations require the essential information to take part in the search and rescue process as soon as possible after an aircraft accident encountered. The amount of survivals, commencing of aircraft accident and investigation process and to acquaint with safety recommendations to avoid similar coincidences will depend upon the accessibility to crash area same time as the investigation of the black box. While implementing a combined system of the fixed black box and a deployable black box SAR organizations and aircraft accident and investigators will be able to commence their duties without any delay as ELT provides the reliable information about the occurred incident via a satellite announcement. And also in a case where, if the permanently fixed black box is being damaged and the process of data recovery is unreachable, the deployable recorders will provide a backup source of evidence which will give hope for the investigators to continue with their responsibility. This research concludes while introducing a new technique to eject the deployable recorders to trace ground with less impact. This is suggested with the mechanism by deploying a parachute at the same time of ejecting the recorder. Another idea recommended is to encounter a manual deploying operating system which can be activated by the pilot, when discharging the deployable recorder. When the pilot is unable to recall to make this decision, then the automatic system should be triggered to eject the deployable black box.

Legal aspects of investigation mainly focus on the provisions of the Annex 13 to the Convention on International Civil Aviation. Its Chapter 3 stipulates the protection of evidence and the responsibility of the State of Occurrence for the custody and removal of the aircraft. The Annex further defines how the State must handle requests for participation in the investigation from other States.

\section{REFERENCES}

ABEYRATNE R. Rulemaking in Air Transport: a Deconstructive Analysis. Cham, Springer. 2016; 97-99.

ABEYSEKARA TB. A Proposal for the Protection of Digital Databases in Sri Lanka (PhD Thesis, University of Exeter-UK 2013) available at <https://ore.exeter.ac.uk /repository/handle/10871/14172> . Accessed: 27 November 2016.

AUSTIN P ROBERT. National Transportation Safety Board, International Transportation Safety Association 1999, 'The Use of Deployable Flight Recorders in Dual Combi Recorder Installations', Transportation Recording: 2000 and beyond. International Symposium on Transportation Recorders, NTSB, Arlington, Virginia.

BHATTACHERJEE A. Social Science Research: Principles, Methods, and Practices. Textbooks Collection. Book 3. 5. 2012. 
http://scholarcommons.usf.edu/oa_textbooks/3. Accessed: 30 November 2016.

BIEHLER G. Property Rights for Individuals under International Humanitarian Law. 45. Bd., No. 3 Archiv des Völkerrechts. 2007; 432-441.

BONSOR K \& CHANDLER N. How black boxes work. Available at: http://science.howstuffworks.com/transport/flig fl/modern/black-box.htm. 2001. Accessed: 26 November 2016.

BUREAU ATS. Black box flight recorders. Available at: https://www. atsb.gov.au/publications/2014/black-box-flightrecorders/. Accessed: 27 November 2016.

FEDERATION $\mathrm{OF}$ AMERICAN SCIENCETISTS \& PIKE J. Ejection seats. Available at: http://fas.org/man/dod101/sys/ac/equip /eject.htm. 1999. Accessed: 25 November 2016.

FLYING L. Flight operations, emergency Locator transmitter, Langley flying school. Available at: http://www.langley flyingschool.com/Pages/Flight\%20OperationsEmergency\%20Locator\%20Transmitter.html. 2016. Accessed: 25 November 2016.

FOUNDATION FS. 'Flight Safety Foundation White Paper', Deployable Flight Recorder Systems. 2014; 1-2.

ICRC. What is International Humanitarian Law? Advisor Service on International Humanitarian Law. 2004; 1.

LANZOTTI D \& FERGUSON D. 'Databases and The Law' (This paper was prepared for Prof. Laura Gasaway's Cyberspace Law course at the UNC School of Law for Spring. 2006. Available at, $<$ http://www.unc.edu/ courses/2006spring/law/357c/001/projects/doug f/node1.html> Accessed 26 November 2013.
LBLAKE VAN DEN HEUVEL. DRS Technologies Canada Ltd. 'Automatic Deployable Flight Recorders (ADFRs)', Emerging Flight Data And Locator Technology, National Transportation Safety Board, Washington. 2014.

MÉDECINS SANS FRONTIÈRES. The Practical Guide to Humanitarian Law. 2016. Available at: http://guide-humanitarianlaw.org/content/ article/3/protected-objectsand-property/ Accessed on: 29 November 2016.

MILLARD C. 'Communications Privacy' in Ian Walden and John Angel (eds), Telecommunications Law and Regulation (2nd edn, OUP. 2005; 369-381.

WERFELMAN L. 'Thinking Outside the (Black) Box', Aero Safety WORLD. 2009; 4(8): 24-27. 